\title{
Innovación en producto y método comercial en el sector panificador: Lecciones de las Mipymes de Cali, Colombia, 2018
}

\author{
Product innovation and market and the bakery industry: \\ experience from SMEs in Cali, Colombia, 2018
}

DOI: $10.22458 /$ rna.v12i 1.3256

\author{
Msc. Iris María Vélez Osorio ${ }^{1}$ \\ ${ }^{1}$ Fundación Universitaria Católica Lumen Gentium, Departamento de Administración, Cali, Valle del Cauca, \\ Colombia; irismariavelez@gmail.com, https://orcid.org/0000-0003-2532-3335
}

\section{RESUMEN}

Esta investigación se enfoca en la innovación de productos y en el método comercial para la industria de la panificación donde la mayoría de las empresas crean innovaciones incrementales, tanto de mercado como de productos. El tipo de investigación es cualitativo. Se estudiaron 36 pymes mediante la técnica de la entrevista cuyo contenido fue codificado y analizado. A partir de allí, se encontraron tres elementos: A) la innovación de producto se vincula al contexto geográfico de acuerdo con las características socioeconómicas de la ciudad y cambios en el clima; B) el sector panificador favorece innovaciones de mercadeo, particularmente de distribución mientras desarrolla estrategias de integración vertical para llegar a lugares geográficos donde establecer un punto de venta más costoso; C) las innovaciones son creadas por los panaderos más que por los propietarios, lo cual tiene implicaciones en el crecimiento de la empresa.

\begin{abstract}
This research focuses on product innovation and marketing method for the bakery industry, where most of the companies may create gradual innovations, both in terms of marketing and products. This research of a qualitative type involves 36 different SMEs that were studied by means of interviews, whose content was codified and analyzed. As a result, three elements were found: A) product innovation is linked to the geographical context according to the city socio-economic characteristics and climate changes; B) the bakery industry promotes marketing innovation, particularly distribution, while developing vertical integration strategies to reach geographical places where a more expensive outlet may be set up; C) innovations are rather created by the bakers than by the owners, which has implications for the company's growth.
\end{abstract}

\section{RÉSUMÉ}

Cette recherche se centre sur l'innovation de produits et la méthode commerciale dans l'industrie boulangère, où les entreprises créent des innovations graduelles, tant de marché que de produits. Cette recherche de type qualitatif implique 36 PME qui ont été étudiées au moyen d'entrevues, dont le contenu a été codifié et analysé. Il en résulte trois éléments : A) l'innovation de produits est liée au contexte géographique selon les caractéristiques socio-économiques de la ville et les variations du climat ; B) le secteur de la boulangerie favorise l'innovation dans la méthode commerciale, particulièrement la distribution, tout en développant des stratégies d'intégration verticale pour atteindre des lieux géographiques où un point de vente plus cher peut être établi; C) les innovations sont davantage créées par les boulangers que par les propriétaires, ce qui a des implications dans la croissance de l'entreprise.

\section{RESUMO}

Esta investigação é enfocada na inovação de produtos e no método comercial para a indústria de panificação, onde a maioria das empresas cria inovações incrementais, tanto de mercado quanto de produto. O tipo de investigação é qualitativo. 36 PMEs foram estudadas através da técnica de entrevista, cujo conteúdo foi codificado e analisado. A partir disso, foram encontrados três elementos: A) a inovação de produto está vinculada ao contexto geográfico de acordo com as características socioeconômicas da cidade e as mudanças no clima; B) o setor de panificação favorece inovações de marketing, particularmente distribuição, ao mesmo tempo em que desenvolve estratégias de integração vertical para alcançar localizações geográficas onde estabelecer um ponto de venda mais caro; C) As inovações são criadas pelos padeiros mais do que pelos proprietários, tendo implicações para o crescimento da empresa.

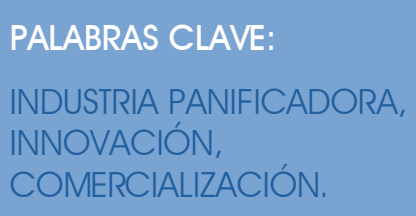

PALABRAS CLAVE:

INDUSTRIA PANIFICADORA,

INNOVACIÓN,

COMERCIALIZACIÓN.
KEYWORDS:

BAKERY INDUSTRY,

INNOVATION,

MARKETING.
MOTS-CLES:

INDUSTRIE BOULANGÈRE,

INNOVATION,

COMMERCIALISATION.
PALAVRAS-CHAVE:

INDÚSTRIA DE

PANIFIÇÃO, INOVAÇÃO,

COMERCIALIZAÇÃO. 


\section{INTRODUCCIÓN}

La industria panificadora ha sido expuesta a nuevas tendencias, en particular, aquellas que requieren cambios completos en insumos, mejores técnicas, métodos y tecnología. Algunos retos para el sector panificador provienen del contexto, del entorno cultural y geográfico en particular donde los consumidores demandan productos saludables y con ingredientes naturales; en consecuencia, las mipymes del sector deben crear innovaciones en productos, servicios, procesos (de Albuquerque Lima y Lauria, 2018) y métodos comerciales (Bigliardi Galanakis, 2020; Manual de Oslo, 2005) que cumplan con estas expectativas y les permita ampliar su grupo de clientes, en el método comercial por ejemplo, la mejora de empaques, nuevos canales de ventas y formas de promoción, son algunas de las prácticas más usadas en esta forma de innovación.

Durante los últimos 10 años, el sector panificador ha modificado progresivamente el tipo de productos que ofrece, dada la aparición de un consumidor informado y con grandes preocupaciones respecto a la salud, el peso y la alimentación consciente. Estas tendencias estudiadas de una manera amplia en revistas académicas dedicadas a los alimentos (Grimsby y Kure, 2019; Schoen, 2017) han demostrado que el consumidor (Dettori et al., 2020) es la principal fuente de información para el desarrollo de nuevos productos. Por tanto, convertir una preocupación en un producto, que luego será una necesidad constante del mercado parece ser la estrategia comercial más usada en la industria panificadora colombiana.

Algunos expertos reconocen que el desarrollo de nuevos productos (Sandhu et al., 2020), (Bijlwan et al., 2020) en el sector panificador se fundamenta en productos de granos enteros, con menos azúcar y artesanales con ingredientes frescos. Estas formas de productos innovadores se han complementado con una nueva manera de ser comercializados directamente a la puerta del consumidor y a partir de intermediarios como tiendas especializadas en recursos saludables y aplicaciones para envíos.

Reconocer estos cambios en el sector permite, además, entender que el sector panificador no solo está en constante crecimiento (Fernandes, et al., 2020) sino que ha optado por especializarse en productos diferenciados que ofrecen una experiencia de consumo distinta y por tanto, crean más valor para el consumidor lo cual en un futuro modificará en su totalidad tanto el proceso productivo (Hullova, Simms, Trott, y Laczko, 2019) como la cadena de suministro del sector.

La innovación en el sector alimenticio cuenta con múltiples formas de innovación, algunas de ellas: innovaciones en método organizativo, innovación en método comercial, innovación social, innovación ambiental, innovación en producto, innovación en proceso productivo. así como inversiones en investigación y desarrollo así como el uso de tecnologías (Navyashree y Bhat, 2020, Mizanbekova, Bogomolova, y Bogomolov, 2017) que permiten un desarrollo mejorado tanto de sus insumos, procesos y productos; la problemática radica, sin embargo, en la posibilidad de innovar para las organizaciones más pequeñas.

En el sector panificador colombiano, en particular dentro de la ciudad de Cali, lo componen en su mayoría compañías mipymes las cuales no siempre cuentan con recursos económicos, recursos humanos, información y tecnología para innovar (de Waal, Tiwari, y McMurray, 2018). En La mayor parte de la innovación se fundamenta en esfuerzos aislados por desarrollar productos y servicios que respondan a los requerimientos de los clientes (Nwewi et al., 2017), en otros casos, a tendencias del sector que presionan a la compañía a innovar, pero no a un esfuerzo planeado por hacerlo.

El objetivo de la investigación es determinar las formas de innovación que predominan en el sector, sus causas y barreras a la innovación. En particular, se establece el análisis de esta industria por ser representativa de la ciudad de Cali donde ocurre el estudio y el cual genera un gran impacto económico y social. Para realizarlo se aplica una metodología cualitativa que permite enriquecer el análisis y entender de manera directa la perspectiva de los gestores de la industria, cómo y de qué forma su quehacer empresarial les permite innovar, así como los impedimentos que han encontrado al hacerlo. 


\section{METODOLOGÍA}

Esta investigación a través de una metodología cualitativa, aplicó entrevistas con administradores, propietarios, empleados de las empresas mipymes panificadoras en la ciudad de Cali, Colombia, cuya elección se debe a que la ciudad cuenta con una amplia cultura gastronómica derivada del pan y variedad de productos relacionados.

Con el fin de contar con una muestra representativa del sector se realizó muestreo probabilítico estratificado, a partir de los estratos socioeconómicos (del 1 al 5) en los que se encontraban las panaderías, para obtener un intérvalo de 4 a 7 panaderías por estrato socioeconómico.

En caso que alguna de las organizaciones no accedieran a realizar la entrevista, se buscaba otra organización en el sector socioeconómico, un total de 36 entrevistas fueron realizadas como muestra representativa del sector cuyos detalles se presentan en la tabla número 1.

El instrumento de recolección de datos contó con cuatro apartados; el primero, de contex to para entender la estrategia de innovación de la compañía; el segundo, en relación a los recursos humanos, la tecnología y la innovación; el tercero, directamente asociado con los tipos de innovación; el cuarto, en relación con las barreras a la innovación, entre ellos la inversión y financiación, los competidores y el conocimiento frente a la innovación.

Las entrevistas tuvieron lugar durante el año 2018. Antes de realizar cada entrevista y con el fin de obtener información completa y respuestas sólidas, se realizó un consentimiento informado por cada una, se trabajó a partir del método cualitativo desde un análisis de contenido y codifcación de datos.

\section{Tabla 1}

Detalles de la Muestra

\begin{tabular}{l|c}
\multicolumn{1}{c|}{ Entrevistados } & Número de entrevistados \\
\hline Propietarios & 5 \\
\hline Administradores & 22 \\
\hline Personal de Apoyo & 9
\end{tabular}

Fuente: elaboración propia de acuerdo con datos recolectados.

Para realizar el análisis se parte de esta proposición: existen distintas formas de innovación en el sector panificador, ellas dependen de los requerimientos de los clientes (Busse y Siebert, 2018) y las tendencias del sector.

Para responder a esta proposición, el análisis cualitativo contó con preguntas abiertas en varias categorías, entre ellas:

1.) Tipos de Innovación

2.) Inversión en Innovación

3.) Barreras a la Innovación

Además, el análisis de contenido se realizó de forma manual, todas las transcripciones de las entrevistas fueron codificadas por números en lugar de los nombres de los propietarios, administradores o empleados entrevistados con el fin de evitar sesgo en en el análisis.

Cada entrevista, como unidad de análisis, fue leída y analizada a profundidad, para construir las categorías a partir de códigos que permitieron establecer los resultados. 


\section{RESULTADOS}

Primero se presentan las categorías de análisis; luego los códigos que se refieren a los datos encontrados y algunos ejemplos de los apartados de texto de las entrevistas que dan cuenta de las categorías y códigos los cuales se utilizaron para entender las formas de innovación del sector (tablas 2 y 3); luego se explican los tipos de innovación encontrados y sus aplicaciones; finalmente, se presentan las proposiciones para resultado del análisis.

\section{Tabla 2}

Códigos para la Categoría de Innovación en Producto y Servicios

\section{Codificación}

\begin{tabular}{l}
\hline Diversidad, insumos integrales, línea fitness, hábitos de consumo, productos frescos, menos azúcar, ingredientes naturales, Libre de glúten. \\
\hline "crear nuevos sabores en los panes, como integrales y galletas" \\
\hline "el cliente se aburre de lo mismo" \\
\hline "aquí en Cali al amanecer, la gente come pan, pandebono y buñuelo" \\
\hline "el pan integral es muy apetecido" \\
\hline "las líneas de pasteles de frutas y con menos azúcar, fue la última innovación" \\
\hline "se ofrece servicios novedosos pensando en lo que ofrecen los competidores" \\
\hline "A partir de grupos de proyectos se promueve la innovación" \\
\hline "antes había que hacer un proceso, ahora... los que manejan productos de panadería ya nos entregan el producto casi listo" \\
\hline "las innovaciones en producto las realiza el área de frescos, el gerente" \\
\hline "la tendencia es un consumidor que le gusta lo integral" \\
\hline "los costos de la harina y el azúcar cambian mucho, lo que daña el margen de rentabilidad...hay que buscar alternativas" \\
\hline "los productos con menos azúcar gustan mucho, pero visualmente no son atractivos" \\
\hline "Una cliente nos pidió unos panes con semillas y sin azúcar, tuvimos que aprender con ese encargo" \\
\hline "ahora incursionamos en panes con vegetales, suena raro pero ha gustado, es como una pizza...algo asi" \\
\hline "las innovaciones en producto las realiza el panadero" \\
\hline "En el sector panificador estamos trabajando de una forma muy artesanal y empírica"
\end{tabular}

Fuente: elaboración propia de acuerdo con datos recolectados.

\section{Tabla 3}

\section{Códigos para la Categoría de Innovación en Método Comercial}

\section{Codificación}

Servicio al cliente, distribución, entrega del producto, empleados, cadena de logística, publicidad, empaque, mejoras en la comunicación con el cliente, servicio postventa, impacto ecológico.

\section{Texto}

\begin{tabular}{l}
\hline "lo que el cliente más valora en nuestra organización es la calidad humana en la atención" \\
\hline "cambiar la logística para la entrega de los pedidos, para que al cliente le lleguen más rápido y más puntual" \\
\hline "Se realizan mejoras en ventas a través de aplicaciones (apps) para vender por internet" \\
\hline "la organización realiza mejoras en las área de mercadeo y ventas, especialmente publicidad" \\
\hline "los clientes quieren servicio a domicilio" \\
\hline "Se realizan reuniones de asesoramiento sobre el servicio al cliente" \\
\hline "el desconocimiento en el proceso de innovación impide la innovación" \\
\hline "Se trabaja en innovación en medio ambiente"
\end{tabular}




\begin{tabular}{l}
\hline "Las promociones con productos como leche y yogurt, por la compra de pan serían las innovaciones en marketing" \\
\hline "Capacitamos en servicio al cliente al personal" \\
\hline "generalmente se hacen mejoras en la forma de llegar al cliente" \\
\hline "la ubicación estratégica ayuda a mejorar las ventas" \\
\hline "se contratan mercaderistas por días, algunas veces" \\
\hline "el empaque ahora los clientes lo piden de papel y no de plástico" \\
\hline "nos ofrecieron unas bolsas de bagazo de caña" \\
\hline "Algunos clientes piden el domicilio, con empaques reciclables" \\
"Ahora tenemos una política de cero plástico, ha sido difícil pero hacemos el propósito"
\end{tabular}

Fuente: elaboración propia de acuerdo con datos recolectados.

\section{Innovación en producto}

A partir del análisis de las entrevistas realizadas, se han encontrado tres tendencias específicas en el desarrollo de nuevos productos: de granos enteros, con menos azúcar y artesanales con ingredientes frescos.

1. Productos de granos enteros: responde a una necesidad transmitida por los clientes. De acuerdo con las organizaciones entrevistadas, sus consumidores presentan intolerancia al gluten; también, llamada condición celiaca. Esto responde a una innovación incremental, no crean nuevos productos, se modifica la base principal de este, el tipo de harina y otros ingredientes añadidos, sin modificar tipología y gramaje.

Es importante reconocer, de acuerdo con lo expuesto por los gestores del sector panificador, que la principal fuente de información para la innovación proviene de las preferencias del consumidor a partir de la investigación de mercados y los buzones de sugerencias.

Por tanto, establecer este tipo de comunicación con el cliente es la principal fuente para modernizar un sector tan competido como el panificador, especialmente en un contexto local como el caleño que cuentan con casi una panadería por cuadra.

Desde la perspectiva de los stakeholders, las mipymes empresas de la industria logran validar el enfoque de los clientes dentro de sus grupos de interés que esperan un producto adaptado a sus necesidades, especialmente, que permita genera valor asociado a la experiencia de consumo.

2. Productos con menos azúcar: consecuencia de los requerimiento de los clientes; en particular, debido a la tendencia creciente en los últimos años de ser sustituida por otros ingredientes, sin afectar la calidad y sabor de los productos.

Es importante destacar, que este tipo de productos se desarrollan a partir de las propuestas del chef o panadero de la compañía el cual propone algunas innovaciones relacionadas y logra crearlos.

Además, son muy pocas las organizaciones dedicadas exclusivamente a este tipo de innovación, algunos de los proveedores del sector se han volcado a ofrecer insumos distintos (Stevia, agave, miel, frutas, entre otros) e incluir otros (edulcorantes) que permiten lograr el sabor dulce de algunos productos, en especial de repostería, para permitir que algunas pequeñas panaderías se especialicen en la elaboración de productos para diabéticos o sin azúcar para el público en general.

Este tipo de innovación es muy relevante para el sector, dado que, junto con el empleado, permite que haya una construcción de este para responder a las necesidades y gustos de los clientes. Sin embargo, es importante destacar que este tipo de innovación incremental resulta más costosa, los insumos que reemplazan el azúcar pueden implicar costos altos los cuales se trasladan generalmente a los consumidores; en consecuencia, el nicho de mercado de este tipo de productos en la ciudad es pequeño. 
3.Productos artesanales con productos frescos: resulta de gran interés por el hecho de responder a una tendencia del entorno. nace la idea de retornar a los orígenes del sector, modificando el proceso y regresando a insumos frescos, que no aparece en el proceso panificador industrializado, esta innovación es radical para el contexto caleño de este análisis, por lo que es una innovación disruptiva que nace en el entorno y llega a la industria colombiana, se puede considerar como una innovación por imitación.

Es importante destacar que las innovaciones radicales en producto son escasas en el sector; sin embargo, esta forma de innovación para el área panificadora permite crear valor para la industria, pues establece una mejor ventaja competitiva, en especial para aquellas organizaciones que modifican su cadena de valor con el objetivo de lograr ofrecer esta innovación radical de manera rápida, con la ayuda de sus proveedores como aliados para la entrega de mejores insumos más frescos y con mayor calidad.

\section{Figura 1}

\section{Innovación en Producto}

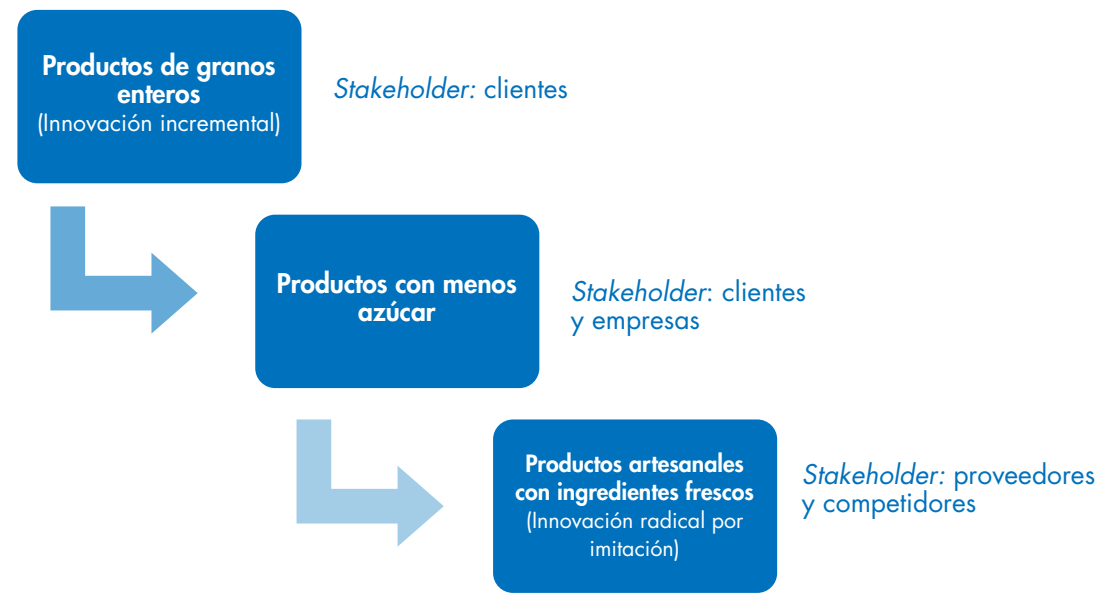

Fuente: elaboración propia de acuerdo con datos recolectados.

\section{Innovación en método comercial}

Desde la innovación en método comercial, que incluye mejoras o prácticas totalmente nuevas en empaques, formas de promoción, canales de ventas, las organizaciones del sector panificador han encontrado una manera de llegar directamente al cliente (Sulistiyani, 2017; Flammini et al., 2017), de acuerdo con las entrevistas realizadas, dos prácticas son comunes entre las panaderías de la ciudad.

Primero, una innovación radical: la distribución móvil surge de la problemática de distancia del usuario y el punto de distribución. En su mayoría, las compañías optaban por contar con domiciliarios que llevarán el producto hasta el cliente; sin embargo, no funcionaba para todos los clientes, pues, algunas zonas alejadas que no tenían un punto de venta, bien sea por ubicación o porque el sector se encontraba en construcción requería que el consumidor observara los productos y pudiera seleccionarlos en vivo y no a través de página web o menú.

El objetivo de los gestores era crear una experiencia positiva para el consumidor, más allá de la venta, el propósito era acercar la marca y sus productos para generar recompra. Dentro de esta práctica, también se incluye para las consumidores que ya conocen el producto y son fieles a la marca, una distribución a través de intermediarios.

La idea anterior refiere a las aplicaciones que permiten el envío desde el punto de venta hasta el consumidor, la ventaja de esta es la posibilidad de crear una experiencia de consumo a través de herramientas digitales y la creación de innovaciones en empaque que permitan la distribución a través de terceros. 
En consecuencia, surge la segunda innovación incremental que corresponde a empaques sostenibles. Las compañías panificadoras han descubierto que el consumidor respeta mucho más a las marcas comprometidas con el medio ambiente, por lo que una disminución en el uso del plástico para los empaques y la creación de alternativas biodegradables da como resultado un retorno al uso artesanal de papel que se ofrecía años atrás; en algunos casos este se deriva del bagazo de caña.

\section{Figura 2}

Innovación en Método Comercial
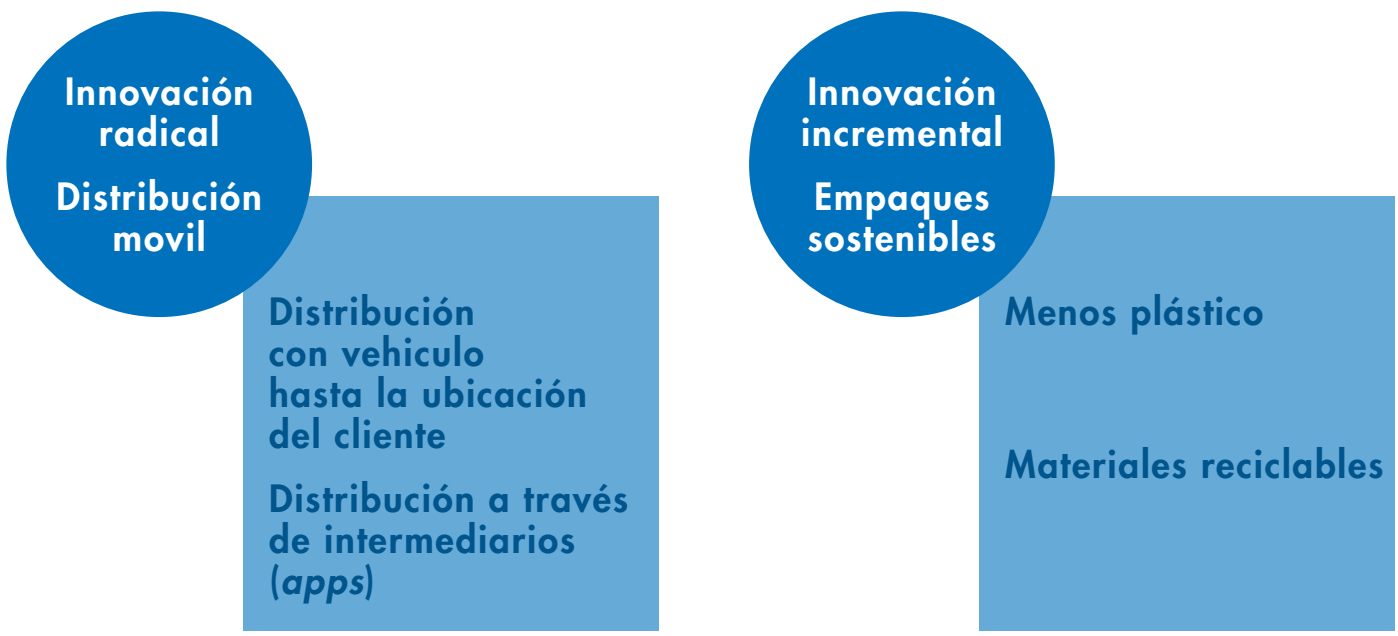

Fuente: elaboración propia de acuerdo con datos recolectados.

De acuerdo con el análisis, se pueden establecer las siguientes proposiciones:

4.) La innovación en el sector panificador se determina a partir de los gustos del consumidor y las tendencias específicas del sector.

5.) La innovación más común es la innovación en producto, seguido de la innovación en método comercial.

6.) La investigación y desarrollo del sector es pequeña donde los panaderos, resposteros y chef, son quienes en general desarrollan las innovaciones.

7.) La principal barrera a la innovación es el desconocimiento del proceso , además de la falta de planeación y recursos exclusivos para hacerlo.

En esta investigación se analizan los tres constructos encontrados: primero, la innovación en producto y sus implicaciones en el crecimiento del sector; segundo la innovación en método comercial; tercero, su impacto en el servicio al ciente. 


\section{CONCLUSIONES}

Las organizaciones panifcadoras se enfocan en innovación de producto e innovación comercial, la primera resulta interesante, debido a las nuevas tendencias en alimentos saludables y los niveles tan competitivos que alcanza el sector en el mercado nacional. Además, esta busca llegar a nichos de mercado pequeños pero exigentes, una propuesta comercial tanto virtual como de distribución puerta a puerta ha tomado fuerza en el sector.

Resulta importante destacar que este estudio, permite entender las formas de innovación y su dinámica en un sector industrial como el panificador, reconoce la innovación en producto y la innovación en método comercial como ejes fundamentales de las prácticas de innovación en el sector.

Como resultado de lo anterior, la industria panificadora ha tenido un éxito creciente,; sin embargo, sería importante establecer en estudios futuros las actividades de innovación que han realizado las compañías del sector para enfrentar los retos de producción y bioseguridad a partir de la situación de la COVID-19 a nivel global.

A partir de los resultados obtenidos se pueden establecer cinco grandes lecciones para los empresarios del sector panificador. La innovación en producto: los requerimientos de los clientes y la escasez de la planeación para la innovación; la innovación en método comercial: las tendencias del sector, la necesidad de mayor inversión en investigación y desarrollo; finalmente, una lección fundamental muy particular del contexto de análisis: el impacto del negocio familiar en la innovación que surge a partir de los diferentes tipos ya estudiados.

En adición, con el análisis realizado, se pueden determinar otras lecciones para el sector panificador, pero en este caso, para las mipymes empresas, estas se clasifican en 5 categorías: los requerimientos de los clientes, las tendencias del sector, escasez de planeación para la innovación, necesidad de mayor inversión en investigación y desarrollo, el impacto del negocio familiar en la innovación.

\section{Los requerimientos de los clientes:}

La primera lección que deja el análisis de innovación en el sector panificador es el cliente como principal fuente de información para la innovación, sus preferencias, estilos y solicitudes deben ser consideradas por la compañía con el fin de llevarlas al desarrollo de nuevos productos y servicios, además de mejorar los existentes.

\section{Las tendencias del sector:}

A nivel global el sector panificador está creciendo y modificando sus métodos y procesos. Es necesario acelerar la lectura por parte del sector panificador colombiano, en particular el caleño, pues la atención a estas tendencias por parte de algunas organizaciones en este ámbito ha permitido una mejora tanto en productos como en el método comercial; sin embargo, un gran cantidad de tendencias en el ámbito tecnológico, social y ambiental no están siendo aplicadas, debido a que en su mayoría las tendencias causantes de la presión generada al sector y modificación en su quehacer son procesos de corto plazo para permitir el desarrollo de innovaciones incrementales, sin una planeación previa de estas innovaciones.

\section{Escasez de planeación para la innovación:}

Las organizaciones del sector panificador en Cali consideran que la planeación estratégica es fundamental y lo hacen a lo largo de sus procesos, pero bajo un planeamiento para la innovación como parte esencial de esta gran estrategia. Aún falta información y preparación para gestionar este proceso. Sin embargo, las compañías son conscientes de la necesidad de iniciarlo a nivel de gestión de la innovación, con información y personal capacitado y responsable para ejecutarlo.

\section{Necesidad de inversión en investigación y desarrollo:}

Los niveles de inversión en investigación y desarrollo son pequeños, en su mayoría corresponde a esfuerzos internos por parte de la organización que en general los lleva a realizar compra de nuevos equipos, maquinaria y herramientas de trabajo, pero no resulta suficiente, estas inversiones tendrían mejores resultados si se complementa con la planeación para la innovación. 


\section{El impacto del negocio familiar en la innovación:}

En el estudio se encuentra presente un elemento fundamental para el sector, la mayoría de las organizaciones son familiares, el sentido de la empresa familiar (Erdogan et al., 2020; Rondi et al., 2020) modifica en particular la manera de innovar. Por tanto, las compañías familiares del sector panificador deben aprovechar las cualidades de la familia para crear proceso de innovación exitoso.

Finalmente, es importante considerar que la innovación en el sector panificador cuentan con importantes retos a futuro, en especial por las implicaciones bien conocidas sobre la salud de algunos productos ofrecidos por el sector.

Por eso, en futuras investigaciones sería importante incluir un estudio longitudinal de las formas de innovación y los efectos de la inversión en investigación y desarrollo. Además, incluir en una medición cuantitativa las variables: sostenibilidad e innovación, stakeholders como fuente de innovación y el efecto moderador del origen familiar de la firma. 


\section{REFERENCIAS}

Bigliardi, B., \& Galanakis, C. (2020). Innovation management and sustainability in the food industry: concepts and models. In The Interaction of Food Industry and Environment (pp. 315-340). Academic Press.

Bijlwan, M., Naik, B., Sharma, D., Singh, A., \& Kumar, V. (2019). Recent developments in dough based bakery products: A mini review. The Pharma Innovation Journal 2019, 8 (5), 654-658.

Busse, M., \& Siebert, R. (2018). The role of consumers in food innovation processes. European Journal of Innovation Management. 21 (1), 20-43.

de Albuquerque Lima, A., \& Lauria, R. L. (2018). Inovação e segurança alimentar em linhas de produção: um estudo de caso em uma indústria de panificação de grande porte/Innovation and food safety in production lines: a case study in a large bakery industry. Brazilian Journal of Development, 4(5), 2600-2615.

Dettori, A., Floris, M., \& Dessì, C. (2020). Customer-perceived quality, innovation and tradition: some empirical evidence. The TQM Journal, 32 (6), 1467-1486.

de Waal, G., Tiwari, R., \& McMurray, A. (2018). Resource-constrained innovation: A viable strategy for firms in the Australian food processing industry. In Governance and Sustainability Conference, Melbourne, Australia.

Erdogan, I., Rondi, E., \& De Massis, A. (2020). Managing the tradition and innovation paradox in family firms: A family imprinting perspective. Entrepreneurship theory and practice, 44(1), 20-54.

Fernandes, C., George, B., \& Mishra, A. (2020). Monginis: A Unique Innovation in the Franchise Model. In Strategies for Promoting Sustainable Hospitality and Tourism Services. IGI Global. 158-170.

Flammini, S., Arcese, G., Lucchetti, M. C., \& Mortara, L. (2017). Business model configuration and dynamics for technology commercialization in mature markets. British Food Journal, 119 (11), 2340 - 2358.

Grimsby, S., \& Kure, C. F. (2019). How open is food innovation? The crispbread case. British Food Journal, 121 (4), 950-963.

Hullova, D., Simms, C. D., Trott, P., \& Laczko, P. (2019). Critical capabilities for effective management of complementarity between product and process innovation: Cases from the food and drink industry. Research Policy, 48 (1), 339-354.

Manual, O. (2005). The measurement of scientific and technological activities. Proposed Guidelines for Collecting an Interpreting Technological Innovation Data, 30.

Mizanbekova, S. K., Bogomolova, I. P., \& Bogomolov, A. V. (2017). Innovation technologies as a factor for increasing the quality of baking industry goods. Food Processing: Techniques and Technology, 45 (2), 142-148.

Navyashree, G. R., \& Bhat, S. (2020). Drivers of ICT investments in bakery and sugar confectionery processed food sub-sector in India. Journal of Agribusiness in Developing and Emerging Economies, 10 (2), 191-202.

Nwewi, H. N., Onwuka, E. M., \& Ogbotubo, E. (2017). Entrepreneurial thinking and competitiveness in bakery industry in Delta State of Nigeria. International Journal of Business, Management and Social Research, 4(01), 192-197.

Rondi, E., Sciascia, S., \& De Massis, A. (2020). Innovation in small family firms. Piccola Impresa/Small Business, (1).

Sandhu, H. K., Sehrawat, R., Kumar, A., \& Nema, P. K. (2020). Overview of Food Industry and Role of Innovation in Food Industry. In Emerging Technologies in Food Science. Springer, Singapore.

Schoen, A. P. (2017). Openness and collaboration in the food sector: mapping the field. British Food Journal, 119 (11), $2493-2506$.

Sulistiyani, S. (2017). Performance Marketing Model through Acculturation Innovation and Market Orientation in the Bakery Industry. Expert Journal of Marketing, 5(1), 1 - 9. 